\title{
Mutations that bypass tRNA binding activate the intrinsically defective kinase domain in GCN2
}

\author{
Hongfang Qiu, Cuihua Hu, Jinsheng Dong, and Alan G. Hinnebusch ${ }^{1}$ \\ Laboratory of Gene Regulation and Development, National Institute of Child Health and Human Development, \\ Bethesda, Maryland 20892, USA
}

\begin{abstract}
The protein kinase GCN2 is activated in amino acid-starved cells on binding of uncharged tRNA to a histidyl-tRNA synthetase (HisRS)-related domain. We isolated two point mutations in the protein kinase (PK) domain, $R 794 G$ and $F 842 L$, that permit strong kinase activity in the absence of tRNA binding. These mutations also bypass the requirement for ribosome binding, dimerization, and association with the GCN1/GCN20 regulatory complex, suggesting that all of these functions facilitate tRNA binding to wild-type GCN2. While the isolated wild-type PK domain was completely inert, the mutant PK was highly active in vivo and in vitro. These results identify an inhibitory structure intrinsic to the PK domain that must be overcome on tRNA binding by interactions with a regulatory region, most likely the $\mathrm{N}$ terminus of the HisRS segment. As Arg 794 and Phe 842 are predicted to lie close to one another and to the active site, they may participate directly in misaligning active site residues. Autophosphorylation of the activation loop was stimulated by $R 794 G$ and $F 842 L$, and the autophosphorylation sites remained critical for GCN2 function in the presence of these mutations. Our results imply a two-step activation mechanism involving distinct conformational changes in the PK domain.
\end{abstract}

[Key Words: eIF2 $\alpha$ kinase; GCN2; regulation; translation; tRNA]

Received January 25, 2002; revised version accepted April 3, 2002.

Protein synthesis can be down-regulated by phosphorylation of Ser 51 in the $\alpha$-subunit of translation initiation factor $2(\mathrm{eIF} 2 \alpha)$. The eIF2 forms a ternary complex with GTP and methionyl-tRNA ${ }^{\text {Met }}\left(\right.$ Met-tRNA $_{i}{ }^{\text {Met }}$ ) that transfers Met-tRNA ${ }_{i}{ }^{\text {Met }}$ to the small ribosomal subunit. Phosphorylated eIF2 inhibits its own guanine nucleotide exchange factor, eIF2B, impeding formation of the ternary complex and subsequent steps in the pathway. The eIF2 $\alpha$ kinase GCN2 is activated by amino acid starvation (Hinnebusch 1996; Harding et al. 2000) and was first identified as a translational inducer of GCN4, a transcriptional activator of amino acid biosynthetic enzymes in budding yeast. Four short upstream open reading frames (uORFs) in the GCN4 mRNA leader underlie a specialized reinitiation mechanism that stimulates GCN4 translation in response to a moderate reduction in ternary complex levels. Thus, GCN2 can induce GCN4, and thereby replenish amino acid pools, without provoking a general shut-off of translation (Hinnebusch 1996).

GCN2 is activated in amino acid-starved yeast cells through binding of uncharged tRNA to a region related to histidyl-tRNA synthetase (HisRS) (Fig. 1A; Wek et al.

\footnotetext{
${ }^{1}$ Corresponding author.

E-MAIL ahinnebusch@nih.gov; FAX (301) 496-6828.

Article and publication are at http://www.genesdev.org/cgi/doi/10.1101/ gad.979402.
}

1989). Mutations in conserved residues involved in tRNA binding by authentic synthetases (the $m 2$ motif) destroyed tRNA binding in vitro and impaired GCN2 function in vivo (Wek et al. 1995; Dong et al. 2000). Starvation for any amino acid activates GCN2 (Wek et al. 1995) and consistently, purified GCN2 bound various uncharged tRNAs with similar affinities, but bound more tightly to uncharged versus charged tRNA ${ }^{\text {Phe }}$ (Dong et al. 2000).

The extreme C terminus of GCN2 (C-term) is also essential for GCN2 function (Wek et al. 1990). The Cterm promotes tRNA binding to the HisRS domain (Dong et al. 2000) and is required for ribosome association in cell extracts (summarized in Fig. 1A; Zhu and Wek 1998). While the C-term, HisRS, and protein kinase (PK) domains all have dimerization activities, only the C-term is critical for dimerization by full-length GCN2 (Qiu et al. 1998, 2001). The conserved $\mathrm{N}$ terminus of GCN2 is the binding domain for the GCN1/GCN20 complex (Garcia-Barrio et al. 2000), a known activator of GCN4 translation. The GCN1/GCN20 complex also has ribosome binding activity (Marton et al. 1997) and may interact with the decoding (A) site on the ribosome. We have proposed that GCN1/GCN20 facilitates the transfer of uncharged tRNA from the A site to GCN2 (Sattlegger and Hinnebusch 2000). 
Qiu et al.

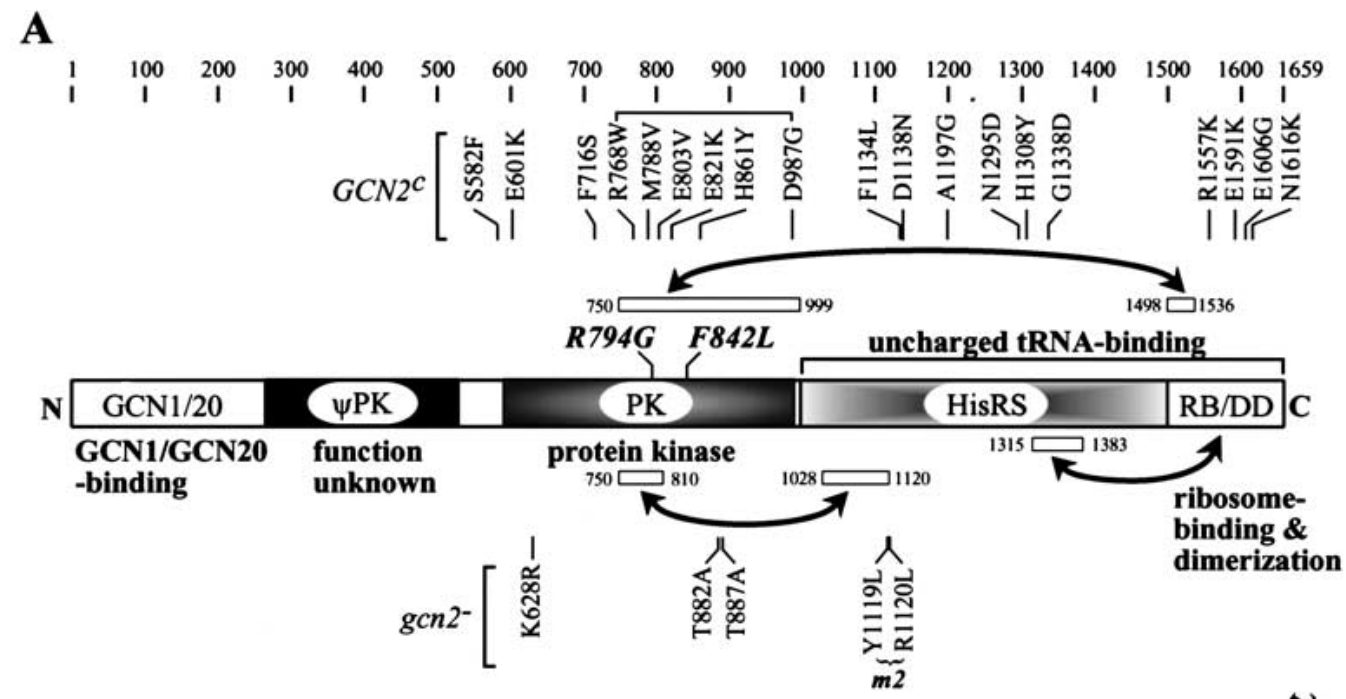

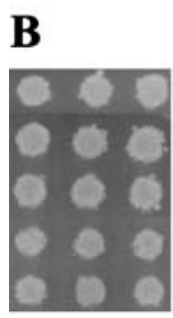

SD

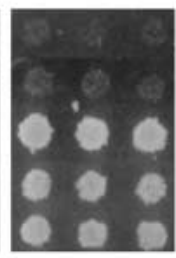

3-AT

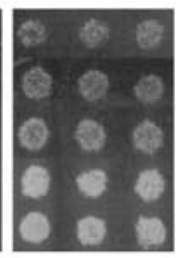

SFT/TRA
Vector

gcn2-m2

GCN2

R794G-m2

$F 842 L-m 2$

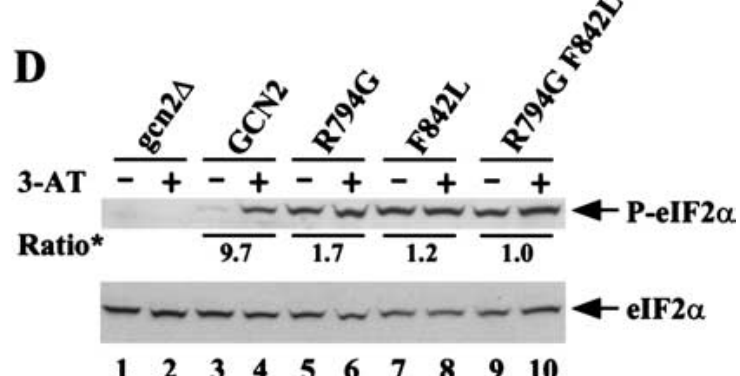

$\begin{array}{llllllllll}1 & 2 & 3 & 4 & 5 & 6 & 7 & 8 & 9 & 10\end{array}$

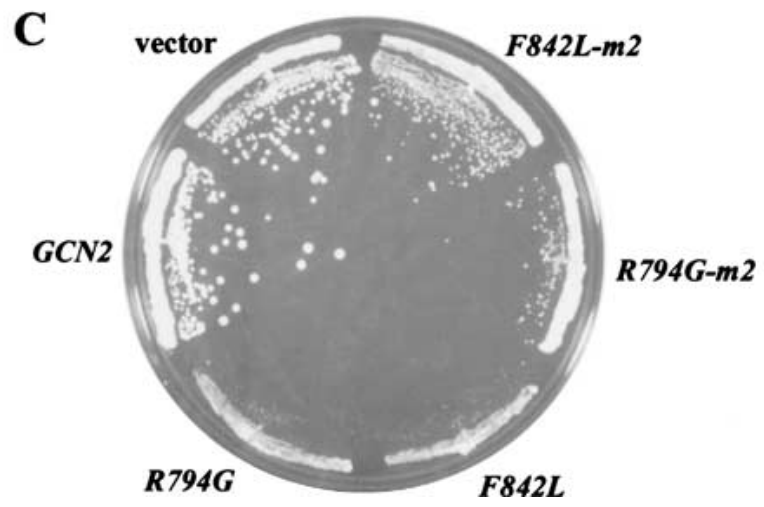

Figure 1. Phenotypes of $G C N 2^{c}$ mutations $R 794 G$ and $F 842 L$. (A) The GCN2 polypeptide of 1659 residues is depicted schematically and divided into the following functional domains: the GCN1/GCN20 binding region (GCN1/20), a degenerate kinase domain ( $\psi \mathrm{PK})$, the protein kinase domain (PK), a histidyl-tRNA synthetase-related region (HisRS), and the C-terminal region, or C-term, required for ribosome-binding and dimerization (RB/DD). The tRNA-binding domain is comprised of the HisRS and C-term segments. Locations of activating mutations $\left(G C N 2^{c}\right)$ are indicated above, whereas selected $g c n 2^{-}$mutations are indicated below the schematic. Segments involved in domain interactions are designated by open boxes connected by arrows. (B) Transformants of gcn $2 \Delta$ strain H1149 carrying empty vector or low-copy plasmids p299 containing gcn2-m2, p722 containing wild-type GCN2, pHQ1096 containing GCN2c $R 794 G$ m2 (R794G-m2), or pHQ1098 containing GCN2 ${ }^{c}-F 842 L-m 2$ (F842L-m2) were replica-plated to SD, SD plus $30 \mathrm{mM} 3-\mathrm{AT}$, or SD plus $0.5 \mathrm{mM} 5$-FT and $0.125 \mathrm{mM}$ TRA (5FT/TRA) and incubated for 3 days at $30^{\circ} \mathrm{C}$. $(C)$ Transformants carrying empty vector, p722, pHQ1102 containing $G C N 2^{c}-R 794 G(R 794 G)$, pHQ1103 containing GCN2 ${ }^{c}$-F842L (F842L), pHQ1096, or pHQ1098 were streaked on an SC-Ura plate and incubated for 3 days at $30^{\circ} \mathrm{C}$. (D) Transformants of gcn2 2 GCD2-K627T strain HQY346 carrying empty vector (gcn24), p722, pHQ1102, pHQ1103, or pHQ1127 containing GCN2 ${ }^{\text {Hyper }}$ (R794G F842L), were grown in SC-Ura-His medium overnight to saturation, diluted into fresh medium at $\mathrm{OD}_{600}=\sim 0.2$, and grown for $6 \mathrm{~h}$ at $30^{\circ} \mathrm{C}$ (odd-numbered lanes). For histidine starvation, 3-AT was added at $10 \mathrm{mM}$ for $1 \mathrm{~h}$ before harvesting (even-numbered lanes). WCEs containing $10 \mu \mathrm{g}$ of protein were resolved by SDS-PAGE on a $4 \%-12 \%$ NuPAGE gel (Novex) and subjected to Western analysis using antibodies specific for eIF $2 \alpha$ phosphorylated on Ser 51 (P-eIF2 $\alpha$, upper panel), or with eIF2 $\alpha$ antibodies (lower panel). The signals on the upper panel were normalized for the corresponding signals in the lower panel, and the ratios of the normalized signals observed in the presence and absence of 3-AT are indicated (Ratio $\left.{ }^{\star}\right)$. 
The isolated C-term domain can physically interact with the HisRS and PK domains (summarized in Fig. 1A). Interestingly, the C-term-PK interaction was weakened by the GCN2 $2^{c}-E 803 \mathrm{~V}$ mutation in the PK domain (Fig. 1A), which confers constitutive activation of GCN2 function in vivo (Qiu et al. 2001). The fact that E803V also increased the affinity of GCN2 for tRNA implied that tRNA binding is impeded by the PK-C-term interaction, such that tRNA binding would normally occur only in starved cells when uncharged tRNA accumulates. The C-term-PK interaction might inhibit PK activity as well, in which case bound tRNA would dissolve this autoinhibitory interaction. The $\mathrm{N}$-terminal portion of the HisRS region (HisRS-N) also interacts with the PK domain (Fig. 1A; Qiu et al. 2001), and the HisRS-N segment stimulates kinase function independently of its role in tRNA binding or dimerization. Thus, it appears that the HisRS-N region remains engaged with the PK domain in the tRNA-bound state to promote kinase activation.

Here we present compelling evidence that the PK domain itself contains an autoinhibitory function. We isolated two mutations in the PK domain ( $R 794 G$ and $F 842 L$ ) as intragenic suppressors of the tRNA binding defect of the $m 2$ mutation in the HisRS domain. Remarkably, these mutations bypass the requirement for all domains flanking the PK moiety for strong kinase activity in vivo and in vitro. The substituted residues are predicted to be close to one another and to the active site in the folded PK domain. Hence, the wild-type residues most likely distort the alignment of active site groups. Our results imply that the PK domain in GCN2 is intrinsically defective and must interact with the flanking HisRS domain on tRNA binding to achieve an active conformation.

\section{Results}

Previously described $\mathrm{GCN}^{\mathrm{c}}{ }^{\mathrm{c}}$ products require the $\mathrm{m} 2$ motif for kinase activity

Point mutations in motif 2 of the HisRS-like domain in GCN2 (gcn2-m2, Fig. 1A) abolish tRNA binding in vitro and inactivate GCN2 function in vivo (Wek et al. 1995). Numerous mutations that activate GCN2 constitutively (GCN2 ${ }^{c}$ alleles) have been isolated in the PK, HisRS-like, and C-term domains (Fig. 1A; Wek et al. 1990; Ramirez et al. 1992). To determine whether these GCN2 ${ }^{c}$ products require tRNA binding for activation, we combined the $m 2$ substitutions with each of the plasmid-borne $G_{C N 2}{ }^{c}$ alleles shown in Figure 1A and analyzed the resulting constructs in a gcn $2 \Delta$ strain. GCN2 function was assessed by measuring growth on medium containing 3-aminotriazole (3AT), an inhibitor of histidine biosynthesis, or on medium containing the amino acid analogs 5-fluorotryptophan (5FT) and 1,2,4-triazolealanine (TRA) (Ramirez et al. 1992). Growth on 3AT medium requires GCN2-dependent derepression of GCN4 and its target genes in the histidine biosynthetic pathway. GCN2 strains are more resistant than wild-type to 5FT and
TRA because they produce constitutively derepressed levels of tryptophan and histidine biosynthetic enzymes (Ramirez et al. 1992).

None of the $G C N 2^{c}-m 2$ mutants grew on 5FT/TRA

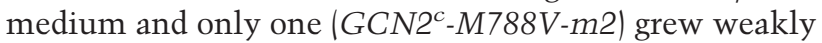
on 3AT medium compared to wild type (data not shown). Hence, the constitutive activation of all previously described GCN2 ${ }^{c}$ proteins is strongly dependent on tRNA binding. This conclusion was confirmed for the GCN2 ${ }^{c}$ $M 788 \mathrm{~V}$ allele by measuring expression of a GCN2-dependent HIS4-1acZ reporter. The GCN2 ${ }^{c}-M 788 \mathrm{~V}-\mathrm{m} 2$ strain showed repressed levels of HIS4-lacZ in nonstarved cells, comparable to that seen in GCN2, gcn2-m2, or gcn2s strains, whereas the parental $G C N 2^{C}-M 788 \mathrm{~V}$ strain had fourfold greater HIS4-lacZ activity (Table 1, rows 1-5).

Mutations R794G and F842L in the PK domain constitutively activate the gen $2-\mathrm{m} 2$ product

We set out to isolate a new class of $G C N 2^{c}$ mutations that would activate GCN2 independently of tRNA binding. As described in Materials and Methods, we identified the R794G and F842L mutations in the kinase domain as strong suppressors of the $3 \mathrm{AT}$-sensitive $\left(3 \mathrm{AT}^{\mathrm{s}}\right)$ phenotype of gcn2-m2. Both the GCN2 ${ }^{c}-R 794 G-m 2$ and $G C N 2^{c}-F 842 L-m 2$ alleles also conferred resistance to 5FT/TRA (Fig. 1B) and derepression of HIS4-lacZ under nonstarvation conditions compared to GCN2 and gcn2$m 2$ (Table 1, rows 2, 3, 6, and 7). They also produced a slow-growth $\left(\mathrm{Slg}^{-}\right)$phenotype on complete (SC) medium (Fig. 1C), characteristic of highly activated $G C N 2^{c}$ alleles (Ramirez et al. 1992). Removing the $m 2$ mutations exacerbated both the $\mathrm{Slg}^{-}$phenotype (Fig. 1C) and derepression of HIS4-lacZ by R794G and F842L (Table 1, rows $6-9)$. Thus, although these mutations greatly reduce the requirement for tRNA binding, restoring motif 2 produced even greater kinase activity.

The allele containing both activating mutations plus $m 2$ conferred a more severe growth defect and greater derepression of HIS4-1acZ than did the GCN2 ${ }^{c}-R 794 G$ $m 2$ and $G C N 2^{c}-F 842 L-m 2$ alleles (Table 1, rows 6-7, 11), showing that $R 794 G$ and F842L have cumulative activating effects. Indeed, combining F842L and R794G in the same allele with wild-type motif 2 produced a lethal phenotype (Table 1, row 10). The latter can be attributed to an intolerably high level of eIF $2 \alpha$ phosphorylation because $G C N 2^{c}-R 794 G-F 842 L$ had no phenotype in a strain expressing nonphosphorylatable eIF $2 \alpha$ (alanine at position 51; data not shown). Henceforth, we refer to $G C N 2^{c}{ }_{-}$ R794G-F842L as GCN2 ${ }^{\text {Hyper }}$ (for hyperactivated GCN2).

We confirmed that the activating mutations produce high-level phosphorylation of eIF $2 \alpha$ under nonstarvation conditions. Because GCN2 $2^{\text {Hyper }}$ is lethal in the presence of wild-type eIF $2 \alpha$, we analyzed a mutant strain where eIF2B is resistant to inhibition by phosphorylated eIF2 because of a mutation in its $\delta$-subunit (GCD2-K627T; Pavitt et al. 1997). Using antibodies that recognize eIF2 $\alpha$ phosphorylated on Ser 51 (P-eIF2 $\alpha$ ) to probe whole cell extracts (WCEs), we found that eIF2 $\alpha$ was phosphorylated in the $G C N 2^{c}-R 794 G, G C N 2^{c}-F 842 L$, and GCN2 $2^{\text {Hyper }}$ 
Table 1. Phenotypic analysis of the GCN2 ${ }^{\mathrm{c}}-\mathrm{R} 794 \mathrm{G}$ and $\mathrm{GCN} 2^{\mathrm{c}}-\mathrm{F} 842 \mathrm{~L}$ mutations ${ }^{a}$

\begin{tabular}{|c|c|c|c|c|c|c|}
\hline & Plasmid & Alleles & $\begin{array}{l}\text { Growth } \\
\text { on SC }\end{array}$ & $\begin{array}{l}\text { Growth } \\
\text { on 3-AT }\end{array}$ & $\begin{array}{l}\text { HIS4-lacZ expression } \\
\text { (Units of } \beta \text {-gal activity) }\end{array}$ & $\begin{array}{c}\text { Relative GCN2 } \\
\text { protein level }\end{array}$ \\
\hline 1 & pRS316 & Vector & +++ & - & 160 & None \\
\hline 2 & p722 & GCN2 & ++++ & +++ & 170 & 100 \\
\hline 3 & p299 & gcn2-m2 & +++ & - & 160 & ND \\
\hline 4 & p912 & $G C N 2^{c}-M 788 \mathrm{~V}$ & ++++ & +++ & 670 & 90 \\
\hline 5 & pHQ928 & $G C N 2^{c}-M 788 V-m 2$ & ++++ & + & 160 & 110 \\
\hline 6 & pHQ1096 & $G C N 2^{c}-R 794 G-m 2$ & + & ++ & 820 & 40 \\
\hline 7 & pHQ1098 & $G C N 2^{c}-F 842 L-m 2$ & + & ++ & 850 & 50 \\
\hline 8 & pHQ1102 & $G C N 2^{c}-R 794 G$ & $-1+$ & + & 1170 & 50 \\
\hline 9 & pHQ1103 & $G C N 2^{c}-F 842 L$ & $-1+$ & + & 1160 & 70 \\
\hline 10 & pHQ1127 & GCN2 ${ }^{\text {Hyper b }}$ & Lethal & NA & NA & NA \\
\hline 11 & pHQ1140 & $G C N 2^{\text {Hyper }}-m 2^{b}$ & $+/-$ & ++ & 1170 & 30 \\
\hline 12 & $\mathrm{pB} 9$ & gcn2-T882A & ++++ & + & 160 & 70 \\
\hline 13 & $\mathrm{pB} 10$ & gcn2-T887A & +++ & - & 160 & 50 \\
\hline 14 & pHQ1251 & $G C N 2^{\text {hyper }}-T 882 A^{b}$ & Lethal & NA & NA & NA \\
\hline 15 & pHQ1252 & $G C N 2^{\text {Hyper }}-T 887 A^{b}$ & ++++ & + & 540 & 60 \\
\hline 16 & pHQ1253 & gcn $2^{\text {Hyper }}-T 882 A T 887 A^{b}$ & +++ & - & 230 & 90 \\
\hline
\end{tabular}

${ }^{a}$ Transformants of gcn2s strain H1149 with the indicated plasmids were rated for growth on SC-Ura plates by scoring the average colony size during the transformation experiments. Growth on 3-AT plates was measured by replica-plating and scored qualitatively after $3 \mathrm{~d}$ of incubation. To measure HIS4-lacZ expression, the transformants were cultured in nonstarvation conditions as described in Materials and Methods and WCEs were prepared and assayed for $\beta$-galactosidase ( $\beta$-gal) activity. The results shown are means calculated from three transformants. The $\beta$-gal activity is expressed as nanomoles of $o$-nitrophenyl- $\beta$-D-galactopyronoside hydrolyzed per minute per milligram of protein. The standard errors are less than $20 \%$. The relative protein levels were determined by Western blot analysis of WCEs using GCN2 antibodies. The Western blot signals were quantified with a scanner (Silverscanner III) and NIH image software (version 1.61). ND, Not determined; NA, not applicable.

${ }^{\mathrm{b}} G C N 22^{\text {Hyper }}$ represents GCN2 ${ }^{c}-R 794 G-F 842 L$.

strains under nonstarvation conditions, whereas wildtype cells contained an appreciable amount of P-eIF2 $\alpha$ only under starvation conditions (Fig. 1D).

\section{The GCN2 $2^{\text {Hyper }} P K$ domain is active in the absence of flanking regulatory domains}

We wished to determine whether the GCN2 ${ }^{\text {Hyper }}$ mutations overcome the requirement for the entire HisRS domain, as well as the ribosome-binding and dimerization functions of the C-term and the GCN1/GCN20 binding domain in the $\mathrm{N}$ terminus. To this end, we combined the GCN2 ${ }^{\text {Hyper }}$ mutations with deletions that remove different segments flanking the PK domain, culminating in a construct that contains only the core PK moiety (Fig. 2). The deletions in the constructs marked with asterisks (rows 3, 5, 6, and 8) destroyed GCN2 function when examined in the absence of the GCN2 ${ }^{\text {Hyper }}$ mutations, conferring 3AT-sensitivity in a gcn2 $2 \Delta$ strain even on highcopy plasmids (data not shown). Remarkably, none of the deletions in Figure 2 inactivated kinase function when combined with the GCN2 $2^{\text {Hyper }}$ mutations. The last two alleles complemented the $3 \mathrm{AT}^{\mathrm{S}}$ phenotype, and derepressed HIS4-lacZ expression above the wild-type level, only when introduced into the $g c n 2 \Delta$ strain on high-copy plasmids (Fig. 2). Nevertheless, the functionality of the last construct provides a striking demonstration that the $G C N 2^{\text {Hyper }}$ mutations bypass the need for all regions flanking the PK domain for substantial kinase activity in vivo.

The products of the last three alleles shown in Figure
2 lack the C-term and, thus, should be defective for ribosome binding. To show that the GCN2 $2^{\text {Hyper }}$ mutations did not activate a cryptic ribosome binding activity in the PK domain, we confirmed that GCN2 ${ }^{\text {Hyper }}-\Delta 1536-$ 1659 (row 6) and gcn2- $\Delta 1536-1659$ were equally defective for ribosome binding in WCEs (data not shown). Deletion of the C-term from GCN2 ${ }^{\text {Hyper }}$ also should abolish dimerization. However, we showed previously that the PK domain can dimerize more efficiently when all flanking regions are eliminated, allowing stable PK-PK dimers to form in vivo (Qiu et al. 1998). To determine whether $R 794 G$ and F842L increase kinase activity by stimulating dimerization of the PK domain, we asked whether these mutations increase the formation of heterodimers by LexA- and HA-tagged PK domains coexpressed in the same cells. As shown in Figure 3A (left panel), $15 \%$ of a LexA fusion containing the C-terminal two-thirds of the kinase domain (LexA- ${ }^{-}$PK[720-999]) was specifically coimmunoprecipitated with the HA-tagged PK domain (3xHA-PK[591-1010]). Importantly, the presence of the GCN2 ${ }^{\text {Hyper }}$ mutations in the LexA- and HA-tagged PK domain had no significant effect on the yield of heterodimers (Fig. 3A, right panel). The same conclusion was reached for analogous constructs containing larger PK segments encompassing residues 591-1010 (data not shown). We conclude that the GCN2 ${ }^{\text {Hyper }}$ mutations do not increase kinase activity by stimulating dimerization of the PK domain.

Deleting the GCN1/GCN20 binding domain at the N terminus of $G C N 2^{\text {Hyper }}$ did not eliminate its lethal phenotype (Fig. 2, row 4), suggesting that the GCN2 $2^{\text {Hyper }}$ 


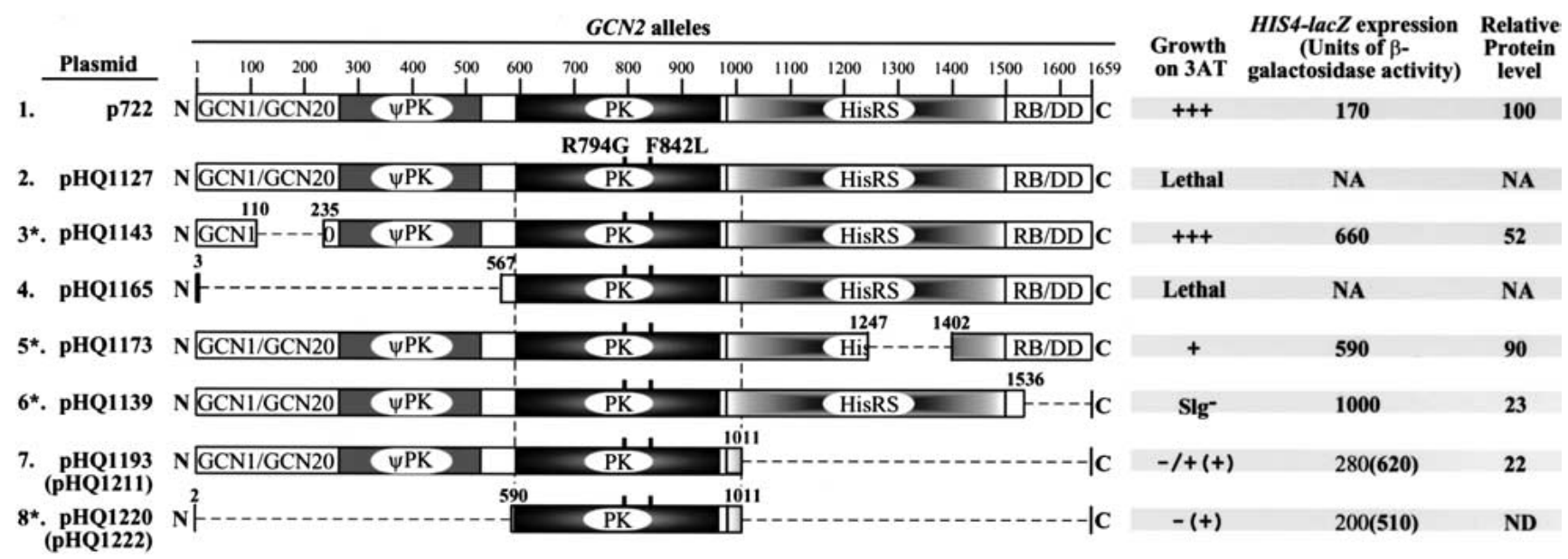

Figure 2. All regions flanking the kinase domain are dispensable for the in vivo kinase function of GCN2 ${ }^{\text {Hyper }}$. The GCN2 alleles are depicted schematically next to the names of low-copy plasmids on which they were introduced into yeast. The names in parentheses designate the corresponding high-copy plasmids. All alleles except the first (wild-type GCN2) contain the GCN2 ${ }^{\text {Hyper }}$ mutations. Deletions are designated by dashed lines, with the amino acid positions of the first and last deleted residues indicated. Transformants of gcn2s strain H1149 were examined for growth on 3-AT medium and HIS4-lacZ expression under nonstarvation conditions as described in Table 1. The last column gives the results of quantitative Western analysis of GCN2 proteins in WCEs analyzed as described in Table 1. The constructs on line 8 differ from all others in containing affinity tags inserted between the start codon and residue 591: Flag and 6xHis for the low-copy plasmid and 3xHA for the high-copy plasmid. Asterisks indicate constructs for which distinct plasmids bearing the identical deletions but lacking the GCN2 ${ }^{\text {Hyper }}$ mutations were tested for complementation of the 3-AT ${ }^{\mathrm{s}}$ phenotype of strain H1149 and found to be indistinguishable from the corresponding empty vector.

mutations bypass the requirements for GCN1 and GCN20 in kinase activation. Confirming this prediction, we found that GCN2 $2^{c}-R 794 G$ and GCN2 ${ }^{c}-F 842 L$ conferred strong 3-AT-resistance in $g c n 1 \Delta, g c n 20 \Delta$, or gcn1s gcn20 strains, whereas the corresponding strains with wild-type GCN2 were $3 \mathrm{AT}^{\mathrm{s}}$. Thus, GCN2 ${ }^{\mathrm{c}}$-R794G and GCN2 ${ }^{\mathrm{C}}$-F842L are highly active in the absence of GCN1 and GCN20. Consistently, the GCN2 $2^{\text {Hyper }}$ allele retained its lethal phenotype in the gcn1s gcn20s strain (data not shown).

The core PK domain containing R794G F842L (PK $\left.K^{\text {Hyper }}\right)$ is active in vitro

Because we could not detect the core PK fragment depicted in Figure 2 (row 8) with GCN2 antibodies, we constructed an allele encoding the same segment tagged at the $\mathrm{N}$ terminus with FLAG epitope under the control of a galactose-inducible promoter. This construct (FL$\left.P K^{\text {Hyper }}\right)$ and the analogous one lacking the $R 794 G$ and F842L mutations (FL-PK) were examined for their effects on cell growth and eIF $2 \alpha$ phosphorylation in a gcn $2 \Delta$ strain. Transformants expressing FLAG-tagged fulllength GCN2 from the GAL promoter (FL-GCN2) or native GCN2 were analyzed in parallel. On medium where the GAL promoter is repressed, all transformants had similar growth rates. On galactose medium, the $F L$ $P K^{\text {Hyper }}$ construct was very toxic, whereas $F L-P K, F L$ GCN2, and GCN2 had no effect on cell growth. The FL-PK $K^{\text {Hyper }}$ construct did not inhibit growth in an isogenic strain expressing nonphosphorylatable eIF2 $\alpha$ (data not shown). Consistently, phosphorylated eIF2 $\alpha$ was produced constitutively in cells expressing FL-PK ${ }^{\text {Hyper }}$ but was undetectable in cells expressing FL-PK or FL-
$\mathrm{PK}^{\mathrm{Hyper}}$-K628R (with a nonfunctional kinase subdomain II) (Fig. 3B, lanes 3-8). As expected, cells expressing FLGCN2 contained P-eIF2 $\alpha$ only under starvation conditions (Fig. 3B, lanes 9 and 10). Thus, the wild-type PK domain $(\mathrm{FL}-\mathrm{PK})$ is completely inactive, whereas FL$\mathrm{PK}^{\mathrm{Hyper}}$ has high-level kinase activity in vivo.

To demonstrate this last point in vitro, we purified the FLAG-tagged proteins and conducted kinase assays using a recombinant form of eIF $2 \alpha(\mathrm{eIF} 2 \alpha-\Delta \mathrm{C})$ and $\left[\gamma^{32} \mathrm{P}\right] \mathrm{ATP}$ as substrates. Phosphorylation of $\operatorname{eIF} 2 \alpha-\Delta \mathrm{C}$ was readily detected using only $5 \mathrm{nM}$ FL-PK ${ }^{\mathrm{Hyper}}$, whereas no P-eIF $2 \alpha-\Delta \mathrm{C}$ was detected in reactions containing 80 -fold more FL-PK (Fig. 3C, lanes 2 and 8). These results prove that the wild-type PK domain is intrinsically defective for eIF $2 \alpha$ phosphorylation and that the R794G and F842L mutations overcome this defect in kinase function. We showed previously that the E803V mutation in the PK domain leads to constitutive phosphorylation of eIF2 $\alpha$ in vivo and increases the tRNA binding activity of GCN2 (Dong et al. 2000). As indicated above, GCN2 ${ }^{\mathrm{c}}$-E803V was inactivated by the $m 2$ mutation, suggesting that E803V activates GCN2 by increasing its affinity for tRNA rather than eliminating an inhibitory structure in the PK domain. Consistently, purified FL-PK ${ }^{\mathrm{E} 803 \mathrm{~V}}$ had barely detectable eIF2 $\alpha$ kinase activity, at least 100 times lower than that of FL-PK ${ }^{\text {Hyper }}$ (Fig. 3C, lanes 13-15 vs. 10-12).

Autophosphorylation sites in the activation loop are required for GCN2 $2^{\text {Hyper }}$ activity

GCN2 autophosphorylates in vitro on two threonine residues in the activation loop of the PK domain, Thr 882 and Thr 887. Alanine substitutions at these positions 
Qiu et al.

Figure 3. The core protein kinase domain containing the GCN2 $2^{\text {Hyper }}$ mutations $\left(\mathrm{PK}^{\mathrm{Hyper}}\right)$ is active in vivo and in vitro. $(A)$ The GCN2 $2^{\text {Hyper }}$ mutations do not stimulate dimerization of the PK domain. Transformants of strain HQY132 containing plasmid pHQ1241 encoding 3xHAPK(591-1010) and either pEG202 (lanes 1-3) or pHQ433 (lanes 4-9) encoding, respectively, LexA alone and LexA-'PK(720999), or containing pHQ1222 encoding $3 \times$ HA-PK $(591-1010)^{\text {Hyper }}$ and either pEG202 (lanes 10-12) or pHQ1235 (lanes 13-18), encoding LexA-'PK(720-999) ${ }^{\text {Hyper }}$, were grown in SC-Ura-His medium to $\mathrm{OD}_{600}=1.2$, and WCEs were immunoprecipitated with HA antibodies. The immune complexes were subjected to Western analysis with antibodies against LexA (upper panels) or HA (lower panels). Lanes labeled I, P, or S contain, respectively, $10 \%$ of the input WCE, $50 \%$ of pellet, and $10 \%$ of the supernatant. Lanes 4-6 and 7-9 depict the results of duplicate experiments, as do lanes 13-15 and 16-18. (B) FL-PK ${ }^{\text {Hyper }}$ phosphorylates eIF $2 \alpha$ in vivo. Transformants of HQY346 containing empty vector pEMBLyex4 (gcn2A), pHQ1213(FL-PK $\left.{ }^{\text {Hyper }}\right)$, pHQ1223 (FL-PK), pHQ1242 (FL-PK Hyper $_{-}$K628R), or pDH103 (FL-GCN2) were grown in SC-Ura-His to saturation, diluted into SCGal-Ura-His at $\mathrm{OD}_{600}=\sim 0.2$ and grown for $7 \mathrm{~h}$ at $30^{\circ} \mathrm{C}$ (-3-AT, odd-numbered lanes), or for $6 \mathrm{~h}$ as just described after which $10 \mathrm{mM}$ 3-AT was added for $1 \mathrm{~h}(+3-\mathrm{AT}$, even-numbered lanes). WCEs were subjected to Western analysis as described in Figure 1D, except that anti-Flag monoclonal antibodies (Sigma) were used to detect the Flag-tagged proteins. (C) $\mathrm{PK}^{\mathrm{Hyper}}$ phosphorylates eIF $2 \alpha$ in vitro. The indicated Flag-tagged core PK proteins were expressed in transformants of strain HQY346 harboring plasmids pHQ1213 (FL-PK ${ }^{\text {Hyper }}$ ), pHQ1223 (FL-PK), or pHQ1261 (FL-PK ${ }^{\mathrm{E} 803 \mathrm{~V}}$ ), grown in minimal galactose medium and purified with anti-Flag affinity resin. The purified proteins were analyzed for kinase activity, at the protein concentrations indicated across the top of each panel, by incubating with $25 \mu \mathrm{M}\left[\gamma_{-}{ }^{32} \mathrm{P}\right] \mathrm{ATP}$ (6000 Ci/mmol, Amersham) and $2.5 \mu \mathrm{M}$ recombinant eIF $2 \alpha-\Delta \mathrm{C}$ purified from E. coli in $20 \mu \mathrm{L}$ kinase buffer for $20 \mathrm{~min}$ at $30^{\circ} \mathrm{C}$. Reactions were stopped by adding $4 \times$ SDS-PAGE sample buffer and boiling, resolved by SDS-PAGE, stained with Coomassie blue (lower panel), and the dried gel was subjected to autoradiography (upper panel). By varying the concentration of eIF $2 \alpha-\Delta \mathrm{C}$ in the assays (data not shown), we verified that saturating amounts of substrate were employed in these assays.

reduced $(T 882 A)$ or eliminated (T887A) GCN2 function in vivo (Romano et al. 1998). As phosphorylation of the activation loop is frequently associated with kinase activation (Johnson et al. 1996), we explored whether the GCN2 ${ }^{\text {Hyper }}$ mutations bypass the requirement for autophosphorylation of Thr 882 and Thr 887 . As shown in Table 1, T887A eliminated the growth defect, but not the 3-AT-resistance and derepression of HIS4-lacZ conferred by GCN2 $2^{\text {Hyper }}$ (rows 10 and 15). Although T882A alone did not suppress the lethality of GCN2 $2^{\text {Hyper }}$, it further inactivated GCN2 $2^{\text {Hyper }}$-T887A, eliminating 3-AT resistance and HIS4-lacZ derepression (Table 1, rows 12-16).
Thus, GCN2 $2^{\text {Hyper }}$ is strongly dependent on both autophosphorylation sites. Consistently, introducing T887A alone or both T882A and T887A into FL-PK $K^{\text {Hyper }}$ eliminated phosphorylation of eIF $2 \alpha$ in vivo, whereas T882A alone led to a small reduction in eIF2 $\alpha$ phosphorylation (Fig. 4A). The fact that $F L-P K^{\text {Hyper }}-T 887 A$ is inactive whereas $G C N 2^{\text {Hyper }}$-T887A retains kinase activity in vivo (Table 1 , row 15 ) is consistent with other results in Table 1 and Figure 2, indicating that the flanking domains enhance PK function in the GCN2 ${ }^{\text {Hyper }}$ protein.

We asked next whether GCN2 autophosphorylation is stimulated by the GCN2 ${ }^{\text {Hyper }}$ mutations. Using antibod- 
A

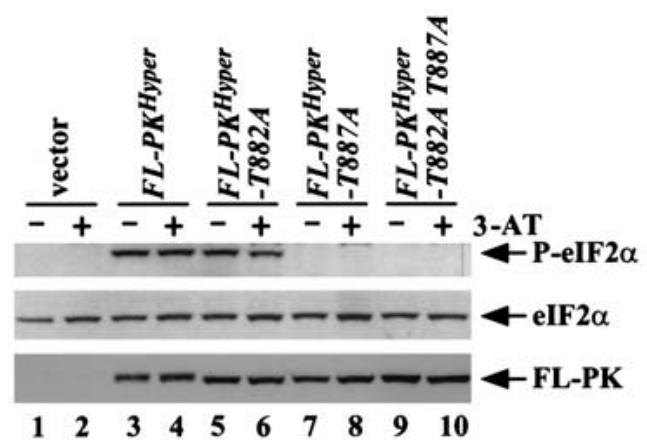

B

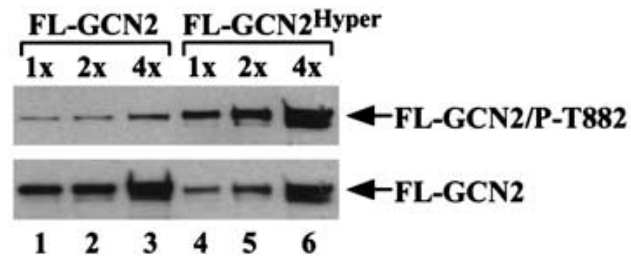

C

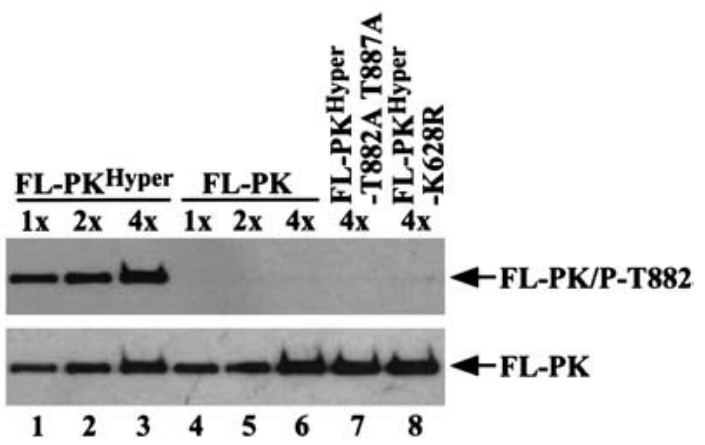

Figure 4. Kinase activation by the GCN $2^{\text {Hyper }}$ mutations is dependent on autophosphorylation of the activation loop. $(A)$ Transformants of HQY346 containing vector pEMBLyex4 (lanes $1-2), \quad$ pHQ1213 (FL-PK $\left.{ }^{\text {Hyper }}\right)$, pHQ1247(FL-PK $\left.K^{\text {Hyper }}-T 882 A\right)$, pHQ1248 (FL-PK $\left.{ }^{\text {Hyper }}-T 887 A\right)$, or pHQ1249 (FL-PK ${ }^{\text {Hyper }}-$ T882A T887A) were analyzed by Western analysis for levels of phosphorylated eIF $2 \alpha$, eIF $2 \alpha$, and the relevant Flag-tagged PK proteins as described in Figure 3B. $(B-C)$ The $G C N 2^{\text {Hyper }}$ mutations increase autophosphorylation of Thr 882. (B) The FL-GCN2 (lanes 1-3) and FL-GCN2 ${ }^{\text {Hyper }}$ (lanes 4-6) proteins were purified as described in Figure 3 using constructs pDH103 and pHQ1131, respectively, and three different relative amounts of each protein $(1 \times, 2 \times$, and $4 \times)$ were subjected to Western analysis using antibodies specific for GCN2 phosphorylated on Thr 882 (upper panel) or Flag antibodies (lower panel). (C) The FL-PK ${ }^{\text {Hyper }}$ (lanes

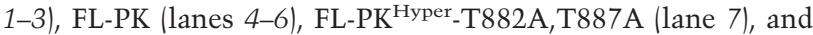
FL-PK ${ }^{\text {Hyper }}$-K628R (lane 8) proteins were purified as described in Figure 3 using constructs pHQ1213, pHQ1223, pHQ1249, and pHQ1242, respectively, and analyzed as in $(B)$.

ies specific for GCN2 phosphorylated on Thr 882 to probe purified GCN2 proteins, we found that FLGCN2 ${ }^{\text {Hyper }}$ contained 10- to 20 -fold more phosphorylated Thr 882 (P-T882) than did FL-GCN2 (Fig. 4B). Consistently, FL-GCN2 ${ }^{\text {Hyper }}$ had $>40$-fold higher in vitro kinase activity than did FL-GCN2 (data not shown). As shown in Figure 4C, P-T882 was readily detected in purified FL-PK ${ }^{\text {Hyper }}$, whereas no P-T882 was found in FLPK. Hence, the wild-type core PK domain is incapable of autophosphorylating Thr 882 in the activation loop, and the GCN2 $2^{\text {Hyper }}$ mutations greatly stimulate autokinase activity. Our findings support the idea that autophosphorylation of the activation loop is a prerequisite for the eIF2 $\alpha$ kinase function of GCN2, even when an inhibitory structure in the PK domain has been eliminated by the GCN2 ${ }^{\text {Hyper }}$ mutations.

\section{Discussion}

Having found that the activating mutation $G C N 2^{c}$ $E 803 \mathrm{~V}$ weakened interaction between isolated PK and C-term segments (Qiu et al. 2001) and also stimulated tRNA binding by GCN2, we proposed that the PK-Cterm interaction interferes with tRNA binding to the HisRS-C-term domains. This would prevent tRNA binding in nonstarved cells where uncharged tRNA concentrations are low. The PK-C-term interaction could also inhibit PK function by an autoinhibitory mechanism that would be released by tRNA binding (Dong et al. 2000). Our finding here that the GCN2-E803V-m2 allele was completely nonfunctional indicates that the weakened interaction between the C-term and PK domains produced by $E 803 \mathrm{~V}$ is not sufficient for kinase activation, and that tRNA binding is still required. In fact, it is possible that the $\mathrm{C}$-term has no autoinhibitory function and that C-term-PK association merely occludes tRNA binding contacts in the C-term. In this view, E803V simply allows tRNA binding and kinase activation to occur at low concentrations of uncharged tRNA. As all other previously described $G C N 2^{c}$ alleles were impaired by the m2 mutation, they may activate GCN2 by this same mechanism.

The GCN2 $2^{\text {Hyper }}$ mutations described here provide a critical new element to our model, as they produce constitutive kinase activation in the absence of tRNA binding. When combined in the same polypeptide, the GCN2 $2^{\text {Hyper }}$ mutations allow the core PK domain to function efficiently under conditions where the wild-type PK domain is completely inert. The simplest explanation for this finding is that the wild-type PK domain is intrinsically defective due to an inhibitory structure involving residues Arg 794 and Phe 842. An important implication of this result is that GCN2 cannot be activated merely by dissociating all flanking regulatory regions from the PK domain; otherwise, the wild-type core PK domain would be active. Rather, the inhibitory structure in the PK domain must be overcome by a stimulatory interaction with another part of the protein.

Based on a sequence alignment of the GCN2 PK domain and protein kinase A (PKA), residues Arg 794 and Phe 842 in GCN2 correspond to Gly 126 and Leu 173 in PKA subdomains V and VIb. Gly 126 in PKA is near the end of the extended chain connecting the $\mathrm{N}$ - and $\mathrm{C}$-terminal lobes of the kinase domain (just prior to helix D), whereas Leu 173 occurs in the middle of $\beta$-strand 7 near the deep cleft between the two lobes (Fig. 5). As the site of catalysis lies within this cleft, both residues are near the active site. In fact, the residue adjacent to Gly 126 in PKA helps in anchoring ATP and the peptide substrate to 


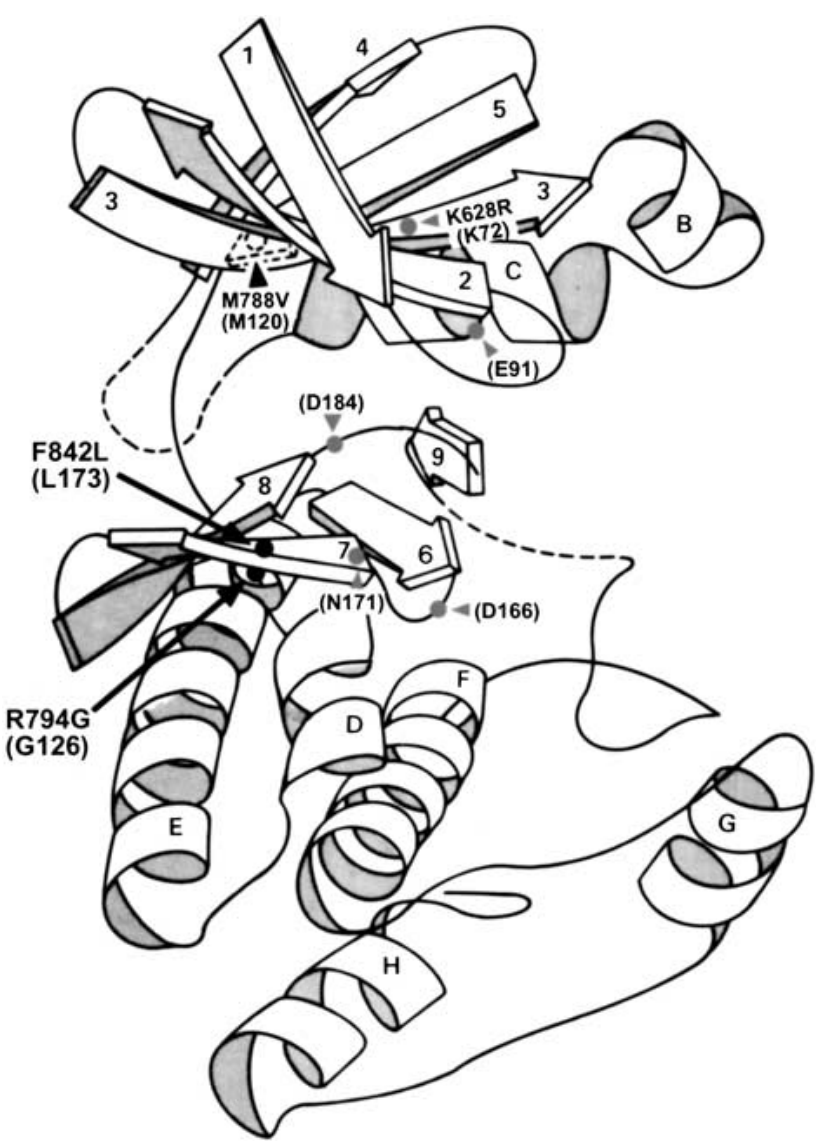

Figure 5. Predicted locations of GCN2 $2^{\text {Hyper }}$ mutations in the folded structure of the protein kinase domain. The three-dimensional structure of the catalytic domain of protein kinase $\mathrm{A}$ is depicted by a ribbon diagram, with $\beta$-strands shown as arrows (numbered from 1 to 9) and $\alpha$-helices shown as coils (labeled from A to H) [adapted from Knighton et al. (1991) and Ramirez et al. (1992)]. The locations of inserts in the catalytic core predicted for GCN2 are shown as dotted lines. The predicted positions of the GCN2 $2^{\text {Hyper }}$ mutations (R794G and F842L) are indicated by black dots. Also shown are the predicted locations of the GCN2 $-M 788 \mathrm{~V}$ and $g c n 2-K 628 R$ mutations, and key residues in PKA required for substrate binding or catalysis. Residue numbers in parentheses are from PKA; those without parentheses are the corresponding residues in GCN2, based on sequence alignments between GCN2 and PKA (Ramirez et al. 1992; Hanks and Hunter 1995b).

the active site, while Leu 173 is only two residues away from an invariant residue in the catalytic loop (Asn 171; Hanks and Hunter 1995b). Computer analysis of PKA (using WebLab ViewerLite 3.2) revealed that Gly 126 and Leu 173 are nearly as close to one another in the folded structure as adjacent residues in the polypeptide chain (Fig. 5). Based on these considerations, we predict that Arg 794 and Phe 842 play a direct role in distorting the GCN2 active site. Consistently, the GCN2 $2^{\text {Hyper }}$ mutations replace these residues with the exact amino acids (Gly and Leu, respectively) found at the equivalent positions in PKA, an intrinsically active kinase, and in a majority of other protein kinases (Hanks and Hunter 1995a).
The HisRS-N region is required for activation of GCN2 independently of its role in tRNA binding. The HisRS-N segment binds to the PK domain between residues 750 and 810 (Qiu et al. 2001), encompassing the predicted extended connector between the $\mathrm{N}$ - and C-lobes of the PK domain and including the inhibitory residue Arg 794. Thus, an attractive hypothesis is that association of the HisRS-N/tRNA complex with this surface of the PK domain alters the relative orientation of the kinase lobes in a way that overcomes the misalignment of active site groups imposed by wild-type residues Arg 794 and Phe 842.

Phosphorylation of the activation loop is frequently required for kinase activation. It is thought that the phosphorylated residue stabilizes a positively charged cluster and thereby promotes the correct orientation and electrostatic environment of the catalytic aspartate (Asp 166 in PKA; Fig. 5) and the proper relative orientation of the $\mathrm{N}$ - and C-lobes (Johnson et al. 1996). We found that the GCN2 $2^{\text {Hyper }}$ mutations did not bypass the requirement for the autophosphorylation sites in the GCN2 activation loop, as replacement of Thr 887 and Thr 882 with alanine almost completely inactivated GCN2 ${ }^{\mathrm{Hyper}}$. Replacement of Thr 887 alone was sufficient to inactivate the core kinase domain in FL-PK ${ }^{\mathrm{Hyper}}$. Based on these findings, we propose that phosphorylation of the activation loop occurs only after the inhibitory structure of the PK domain has been corrected by interaction with the HisRS-N/tRNA module. Consistently, Thr 882 was phosphorylated in the highly active FL-PK ${ }^{\text {Hyper }}$ protein but not in the inactive wild-type core PK construct. Presumably, autophosphorylation stimulates a critical conformational change in the PK domain beyond that produced by association with the HisRS-N/tRNA module. This model has similarities with a mechanism proposed for activation of CDK2 by cyclin, in which binding of cyclin A realigns the active site residues and relieves the steric block to substrate binding imposed by the PSTAIRE helix and activation loop. Phosphorylation of the activation loop is additionally required for full activation of CDK2 (Jeffrey et al. 1995; Johnson et al. 1996).

The fact that the GCN2 $2^{\text {Hyper }}$ mutations bypass the requirement for the $\mathrm{N}$-terminal GCN1/GCN20 binding domain and the C-term, in addition to the HisRS domain, suggests that all of these domains are required for activation of GCN2 by uncharged tRNA. This conclusion supports our proposal that the GCN1/GCN20 complex interacts with the $\mathrm{N}$ terminus of GCN2, anchored to the ribosome through the $\mathrm{C}$-term, to mediate transfer of uncharged tRNAs from the decoding site on the ribosome to the HisRS domain (Sattlegger and Hinnebusch 2000).

\section{Materials and methods}

\section{Isolation of $\mathrm{GCN} 22^{\mathrm{c}}-\mathrm{R} 794 \mathrm{G}$ and $\mathrm{GCN} 2^{\mathrm{c}}-\mathrm{F} 842 \mathrm{~L}$}

GCN2 plasmid pHQ644 was subjected to random mutagenesis in E. coli mutator strain XL1-Red (Strategene). BspEI-Asp718 
fragments were isolated from the mutagenized pHQ644 and used to replace the corresponding fragment of plasmid p299 containing the gcn2-m2 allele (library I). Similarly, the Eco47IIINheI fragment of plasmid p299 was replaced with the corresponding fragments from mutagenized pHQ644 to produce library II. The two libraries were screened in gcn $2 \Delta$ strain H1149 for 3 -AT ${ }^{\mathrm{r}}$ transformants. Of $3.4 \times 10^{5}$ transformants obtained with library I, 24 clones showed a plasmid-dependent $3-\mathrm{AT}^{\mathrm{R}}$ phenotype, while no such clones were isolated from library II. Twenty-two of the 24 plasmids isolated from the 3 -AT ${ }^{\mathrm{R}}$ colonies had an A to G mutation at codon 794 (AGA to GGA), producing the $R 794 G$ substitution. The remaining two plasmids contained a $\mathrm{T}$ to $\mathrm{C}$ mutation at codon 842 (TTT to CTT), generating F842L.

All other plasmids used in this work are listed in Table 2, and details of their construction are available on request. Strains H1149 (Wek et al. 1990) and HQY132 (Qiu et al. 1998) were described previously. Strain HQY346 is a GAL2 derivative of GP3299 (Pavitt et al. 1997).

\section{Biochemical methods}

Transformants of HQY346 bearing the appropriate plasmids encoding Flag-, 6xHis-tagged GCN2 proteins were grown in SD medium to saturation, diluted into minimal medium with $2 \%$ galactose $(\mathrm{SGal})$ and grown to $\mathrm{OD}_{600}=3.0$. Affinity purification of the tagged proteins was conducted essentially as described (Dong et al. 2000). In vitro kinase assays (Dong et al. 2000) and assays of HIS4-lacZ expression (Lucchini et al. 1984) were all done as described previously. The enhanced chemiluminescence (ECL) system (Amersham Pharmacia Biotech) was used to detect immune complexes, and Western signals were quantified by video image densitometry using NIH Image 1.61 software. Phosphospecific antibodies against P-eIF2 $\alpha$ (Biosource International) and polyclonal antibodies against eIF2 $\alpha$ (CM-217) (Cigan et al. 1989), antibodies against GCN2 (Romano et al. 1998), and antibodies against LexA (Qiu et al. 1998) were described previously. Flag- or HA-tagged proteins were detected using FLAG (Sigma) and HA antibodies (Roche). Antibodies specific for GCN2 phosphorylated on Thr 882 were produced by Quality Controlled Biochemicals against a synthetic peptide corresponding to the GCN2 activation loop phosphorylated on Thr 882 (in single-letter code: CPGSSDNL[P-T $\left.{ }_{882}\right] \mathrm{SAI}$ ). The antibodies were preabsorbed with the nonphosphorylated peptide and affinity-purified with the phosphorylated peptide. In Western blot analysis, the antibodies reacted with FL-GCN2 (wild type) but not with FL-GCN2-T882A or catalytically inactive FLGCN2-K628R.

Table 2. Plasmids used in this study

\begin{tabular}{|c|c|c|}
\hline Plasmid & Allele & Reference \\
\hline p722 & GCN2, CEN6, URA3 & Wek et al. 1990 \\
\hline p299 & gcn2-m2 in p722 backbone & Wek et al. 1995 \\
\hline p912 & GCN2 $-M 788 \mathrm{~V}$ in p722 backbone & Ramirez et al. 1992 \\
\hline pB9 & gcn2-T882A, CEN4, URA3 & Romano et al. 1998 \\
\hline $\mathrm{pB} 10$ & gcn2-T887A, CEN4, URA3 & Romano et al. 1998 \\
\hline pHQ644 & GCN2, p722 derivative & Qiu et al. 2001 \\
\hline p 630 & high copy GCN2, $2 \mu, R U A 3$ & Wek et al. 1990 \\
\hline pHQ433 & high copy LexA-'PK(720-999) in pEG202 backbone & Qiu et al. 1998 \\
\hline pHQ928 & GCN2-M788V-m2 in p722 backbone & This study \\
\hline pHQ1096 & GCN2-R794G-m2 in p722 backbone & This study \\
\hline pHQ1098 & GCN2-F842L-m2 in p722 backbone & This study \\
\hline pHQ1102 & GCN2-R794G in pHQ644 backbone & This study \\
\hline pHQ1103 & GCN2-F842L in pHQ644 backbone & This study \\
\hline pHQ1127 & GCN2 ${ }^{\text {Hyper }}$ in pHQ644 backbone & This study \\
\hline pHQ1131 & high copy Flag-6xHis-GCN2 ${ }^{\text {Hyper }}$ under $G A L$ promoter in pEMBLyex4 & This study \\
\hline pHQ1139 & GCN2 ${ }^{\text {Hyper }}-\Delta 1536-1659$ in pHQ644 backbone & This study \\
\hline pHQ1140 & GCN2 $2^{\text {Hyper }}-\mathrm{m} 2$ in pHQ644 backbone & This study \\
\hline pHQ1143 & GCN2 ${ }^{\text {Hyper }}-\Delta 110-235$ in pHQ644 backbone & This study \\
\hline pHQ1165 & $G C N 2^{\text {Hyper }}-\Delta 3-567$ in pHQ644 backbone & This study \\
\hline pHQ1173 & GCN2 ${ }^{\text {Hyper }}-\Delta 1247-1402$ in pHQ644 backbone & This study \\
\hline pHQ1193 & GCN2 ${ }^{\text {Hyper }}-\Delta 1011-1659$ in pHQ644 backbone & This study \\
\hline pHQ1211 & high copy $G C N 2^{\text {Hyper }}-\Delta 1011-1659$ in YEplac195 & This study \\
\hline pHQ1213 & high copy Flag-6xHis-PK(591-1010 $)^{\text {Hyper }}$ under GAL promoter in pEMBLyex4 & This study \\
\hline pHQ1220 & Flag-6xHis-PK(591-1010) $)^{\text {Hyper }}$ in p722 backbone & This study \\
\hline pHQ1222 & high copy $3 \times H A-P K(591-1010)^{\text {Hyper }}$ in p630 backbone & This study \\
\hline pHQ1223 & high copy Flag-6xHis-PK(591-1010) under GAL promoter in pEMBLyex4 & This study \\
\hline pHQ1235 & high copy LexA-'PK(720-999) Hyper in pEG202 backbone & This study \\
\hline pHQ1241 & high copy $3 \times H A-P K(591-1010)$ in p630 backbone & This study \\
\hline pHQ1242 & high copy Flag-6xHis-PK(591-1010 $)^{\text {Hyper }}$-K628R under GAL promoter in pEMBLyex4 & This study \\
\hline pHQ1247 & high copy Flag-6xHis-PK(591-1010 $)^{\text {Hyper }}$-T882A under GAL promoter in pEMBLyex4 & This study \\
\hline pHQ1248 & high copy Flag-6xHis-PK(591-1010 $)^{\text {Hyper }}$-T887A under GAL promoter in pEMBLyex4 & This study \\
\hline pHQ1249 & high copy Flag-6xHis-PK(591-1010) ${ }^{\text {Hyper }}$-T882A T887A under $G A L$ promoter in pEMBLyex4 & This study \\
\hline pHQ1251 & GCN2 ${ }^{\text {Hyper }}-$ T882A in pHQ644 backbone & This study \\
\hline pHQ1252 & GCN2 ${ }^{\text {Hyper }}-T 887 A$ in pHQ644 backbone & This study \\
\hline pHQ1253 & GCN2 ${ }^{\text {Hyper }}$-T882A T887A in pHQ644 backbone & This study \\
\hline pHQ1261 & high copy Flag-6xHis-PK(591-1010) ${ }^{E 803 V}$ under GAL promoter in pEMBLyex4 & This study \\
\hline
\end{tabular}




\section{Acknowledgments}

We are indebted to Ron Wek for plasmid p299 and we thank Tom Dever for help with Weblab ${ }^{\mathrm{TM}}$ ViewerLite 3.2, valuable discussions, gifts of antibodies, and critical reading of the manuscript.

The publication costs of this article were defrayed in part by payment of page charges. This article must therefore be hereby marked "advertisement" in accordance with 18 USC section 1734 solely to indicate this fact.

\section{References}

Dong, J., Qiu, H., Garcia-Barrio, M., Anderson, J., and Hinnebusch, A.G. 2000. Uncharged tRNA activates GCN2 by displacing the protein kinase moiety from a bipartite tRNAbinding domain. Mol. Cell 6: 269-279.

Garcia-Barrio, M., Dong, J., Ufano, S., and Hinnebusch, A.G. 2000. Association of GCN1/GCN20 regulatory complex with the conserved N-terminal domain of eIF2a kinase GCN2 is required for GCN2 activation in vivo. $E M B O J$. 19: 1887-1899.

Hanks, S.K. and Hunter, T. 1995a. The eukaryotic protein kinase superfamily. In The protein kinase facts book (eds. G. Hardie and S. Hanks), pp. 7-47. Academic Press, San Diego, CA.

- 1995b. The eukaryotic protein kinase superfamily: Kinase (catalytic) domain structure and classification. FASEB J. 9: 576-596.

Harding, H.P., Novoa, I., Zhang, Y., Zeng, H., Wek, R., Schapira, M., and Ron, D. 2000. Regulated translation initiation controls stress-induced gene expression in mammalian cells. Mol. Cell 6: 1099-1108.

Hinnebusch, A.G. 1996. Translational control of GCN4: Genespecific regulation by phosphorylation of eIF2. In Translational control (eds. J.W.B. Hershey, M.B. Mathews, and N. Sonenberg), pp. 199-244. Cold Spring Harbor Laboratory Press, Cold Spring Harbor, NY.

Jeffrey, P.D., Russo, A.A., Polyak, K., Gibbs, E., Hurwitz, J., Massague, J., and Pavletich, N.P. 1995. Mechanism of CDK activation revealed by the structure of a cyclinA-CDK2 complex. Nature 376: 313-320.

Johnson, L.N., Noble, M.E.M., and Owen, D.J. 1996. Active and inactive protein kinases: Structural basis for regulation. Cell 85: $149-158$.

Knighton, D.R., Zheng, J., Ten Eyck, L.F., Xuong, N.H., Taylor, S.S., and Sowadski, J.M. 1991. Crystal structure of the catalytic subunit of cyclic adenosine monophosphate-dependent protein kinase. Science 253: 407-414.

Lucchini, G., Hinnebusch, A.G., Chen, C., and Fink, G.R. 1984. Positive regulatory interactions of the HIS4 gene of Saccharomyces cerevisiae. Mol. Cell. Biol. 4: 1326-1333.

Marton, M.J., Aldana, C.R.V.d., Qiu, H., Chakraburtty, K., and Hinnebusch, A.G. 1997. Evidence that GCN1 and GCN20, translational regulators of GCN4, function on enlongating ribosomes in activation of the eIF2a kinase GCN2. Mol. Cell. Biol. 17: 4474-4489.

Pavitt, G.D., Yang, W., and Hinnebusch, A.G. 1997. Homologous segments in three subunits of the guanine nucleotide exchange factor eIF2B mediate translational regulation by phosphorylation of eIF2. Mol. Cell. Biol. 17: 1298-1313.

Qiu, H., Dong, J., Hu, C., Francklyn, C.S., and Hinnebusch, A.G. 2001. The tRNA-binding moiety in GCN2 contains a dimerization domain that interacts with the kinase domain and is required for tRNA binding and kinase activation. EMBO $J$. 20: $1425-1438$.
Qiu, H., Garcia-Barrio, M.T., and Hinnebusch, A.G. 1998. Dimerization by translation initiation factor 2 kinase GCN2 is mediated by interactions in the C-terminal ribosomebinding region and the protein kinase domain. Mol. Cell. Biol. 18: 2697-2711.

Ramirez, M., Wek, R.C., Vazquez de Aldana, C.R., Jackson, B.M., Freeman, B., and Hinnebusch, A.G. 1992. Mutations activating the yeast eIF-2a kinase GCN2: Isolation of alleles altering the domain related to histidyl-tRNA synthetases. Mol. Cell. Biol. 12: 5801-5815.

Romano, P.R., Garcia-Barrio, M.T., Zhang, X., Wang, Q., Taylor, D.R., Zhang, F., Herring, C., Mathews, M.B., Qin, J., and Hinnebusch, A.G. 1998. Autophosphorylation in the activation loop is required for full kinase activity in vivo of human and yeast eukaryotic initiation factor 2 a kinases PKR and GCN2. Mol. Cell. Biol. 18: 2282-2297.

Sattlegger, E. and Hinnebusch, A.G. 2000. Separate domains in GCN1 for binding protein kinase GCN2 and ribosomes are required for GCN2 activation in amino acid-starved cells. EMBO J. 19: 6622-6633.

Wek, R.C., Jackson, B.M., and Hinnebusch, A.G. 1989. Juxtaposition of domains homologous to protein kinases and histidyl-tRNA synthetases in GCN2 protein suggests a mechanism for coupling GCN4 expression to amino acid availability. Proc. Natl. Acad. Sci. 86: 4579-4583.

Wek, R.C., Ramirez, M., Jackson, B.M., and Hinnebusch, A.G. 1990. Identification of positive-acting domains in GCN2 protein kinase required for translational activation of GCN4 expression. Mol. Cell. Biol. 10: 2820-2831.

Wek, S.A., Zhu, S., and Wek, R.C. 1995. The histidyl-tRNA synthetase-related sequence in the eIF-2a protein kinase GCN2 interacts with tRNA and is required for activation in response to starvation for different amino acids. Mol. Cell. Biol. 15: 4497-4506.

Zhu, S. and Wek, R.C. 1998. Ribosome-binding domain of eukaryotic initiation factor-2 kinase GCN2 facilitates translation control. J. Biol. Chem. 273: 1808-1814. 


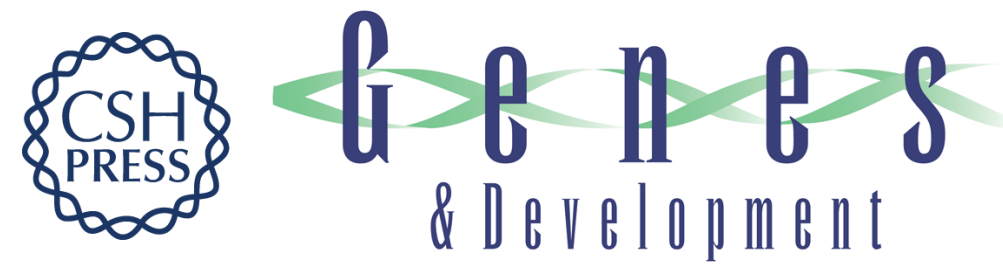

\section{Mutations that bypass tRNA binding activate the intrinsically defective kinase domain in GCN2}

Hongfang Qu, Cuihua Hu, Jinsheng Dong, et al.

Genes Dev. 2002, 16:

Access the most recent version at doi:10.1101/gad.979402

References

This article cites 19 articles, 14 of which can be accessed free at: http://genesdev.cshlp.org/content/16/10/1271.full.html\#ref-list-1

License

Email Alerting

Receive free email alerts when new articles cite this article - sign up in the box at the top Service right corner of the article or click here.

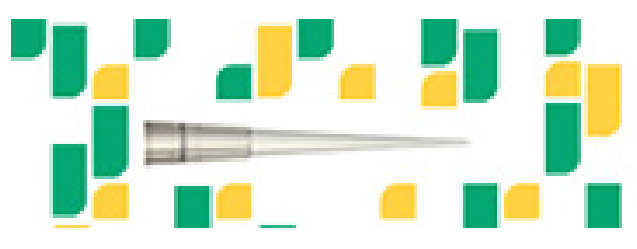

Focused on your science. 